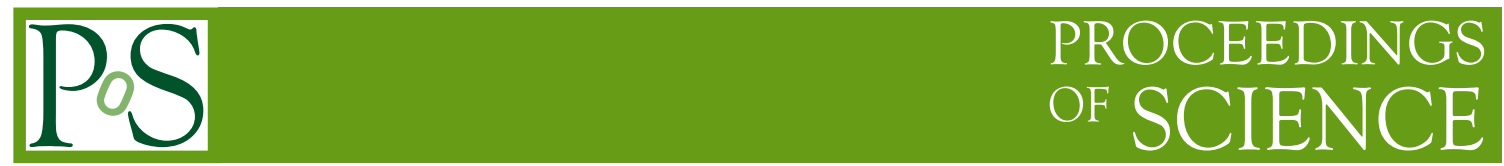

\title{
Studies of open charm and charmonium production at $\mathrm{LHCb}$
}

\author{
E. Polycarpo* \\ Physics Institute/Federal University of Rio de Janeiro, Brasil \\ E-mail: polyeif.ufrj.br
}

On behalf of the LHCb Collaboration

\begin{abstract}
Charm mesons will be abundantly produced in the LHCb experiment. Studies involving open charm and charmonium production are presented in this article, with emphasis on the reconstruction of the final state particles and results from the first runs of data taking, at center of mass energies of 900 and $7000 \mathrm{GeV}$.
\end{abstract}

XVIII International Workshop on Deep-Inelastic Scattering and Related Subjects April 19 -23, 2010

Convitto della Calza, Firenze, Italy

\footnotetext{
*This work has been partially supported by CNPq, FINEP and FAPERJ.

†peaker.
} 


\section{Introduction}

The Large Hadron Collider beauty experiment ( $\mathrm{LHCb}$ ) is one of the four major detectors of the Large Hadron Collider. Its primary goal is to look for indirect evidence of new physics in CP violation and rare decays of heavy flavour hadrons. Although it was designed for the study of bhadrons in nominal LHC operation conditions, when we expect to achieve an integrated luminosity of $2 \mathrm{fb}^{-1}$ in one year of data taking $\left(10^{7} \mathrm{sec}\right)$ at a center of mass energy $\sqrt{s}=14 \mathrm{TeV}$, the operation of the collider with lower luminosity during the long 2010-2011 run at $\sqrt{s}=7 \mathrm{TeV}$ provides excellent conditions for the study of charm decays. The $c \bar{c}$ cross-section is expected to be $\sim 4.7 \mathrm{mb}$ according to PYTHIA, a factor of $\sim 2$ smaller than its value at $14 \mathrm{TeV}$, but still very high. However, the lower event rate allows a tuning of the trigger settings at lower thresholds, thus enhancing the efficiency for prompt charm mesons, including charmonium states.

The spectrometer has a forward geometry covering polar angles in the range $[15,300] \mathrm{mrad}$, due to the predominance of heavy quarks production at small angles with respect to the beam direction. For the final states discussed in this document, the most important subsystems are the VErtex Locator (VELO), the tracking system, the RICH detectors and the Muon Stations (MS).

In the 2009 LHC pilot run, LHCb [1] collected about $6.8 \mu \mathrm{b}^{-1}$ of good data at a center of mass energy of $0.9 \mathrm{TeV}$. This first period was very important for the initial steps of global detector and trigger commissioning and for improvement of the detector calibration and alignment, which started earlier with cosmic particles and events with the beam on targets. Studies of $K_{s}^{0}$ and $\Lambda$ production are also being performed with these data (see [2]). However, not enough data were accumulated to allow the reconstruction of $\mathrm{D}$ mesons and charmonium states. This was only possible after the beginning of the collisions at $7 \mathrm{TeV}$ energy in 2010. One week before the start of this conference, the experiment had collected proton-proton collisions equivalent to an integrated luminosity of $\sim 180 \mu \mathrm{b}^{-1}$.

\section{Open Charm Studies}

The ratio between charm and anti-charm hadrons will be one of the first measurements of the LHCb experiment and will also be a crucial step towards the primary goal which is the measurement of the absolute production cross-sections of $D^{0}, D^{+}, D^{*}+, D_{s}^{+}$and $\Lambda_{c}$ in different rapidity and transverse momentum ranges. All these measurements will also be of great relevance for the tuning of the LHCb MC simulation. With the high statistics provided in the long 2010-2011 run, topics like charm mixing, $\mathrm{CP}$ violation and rare $\mathrm{D}$ decays will also be covered. Charm decays appear as an interesting laboratory for indirect search of New Physics effects in $\mathrm{CP}$ violation studies due to the small levels of the Standard Model predictions. Evidences for charm mixing have been found recently by BABAR, BELLE and CDF. For recent results on charm physics from the B factories, see [3].

Charm mesons and baryons can be produced promptly at the p-p collision or through the decays of b-hadrons. At $\sqrt{s}=7 \mathrm{TeV}$, the PYTHIA expectation for the ratio between the cross-sections for $c \bar{c}$ and $b \bar{b}$ is around 7. For this reason, the current efforts of the Collaboration are concentrated on the study of prompt production. D mesons fly on average $3 \mathrm{~mm}$ from the interaction point, which can be compared to the average flight distance of $7 \mathrm{~mm}$ for B mesons. To be able to exploit 
the prompt charm production, the trigger had to be reoptimized for the simultaneous detection of charm and beauty decays.

The LHCb trigger is divided into two levels. The first level (L0) is a hardware trigger which selects muons, hadrons, electrons or photons with high transverse momentum $\left(p_{T}\right)$. In the high level trigger (HLT), there are two steps: L0 candidates are confirmed with more refined information (HLT1) and the whole event is reconstructed using both inclusive and exclusive selections (HLT2). By lowering all thresholds ( $p_{T}$, impact parameters, etc) the charm yields are estimated to improve a factor 4 compared to the settings optimized for B physics in nominal conditions. The L $0 \times$ HLT 1 efficiency for $D^{*} \rightarrow \pi D^{0}(h h)$ is about $70 \%$. For the data taken so far, the interaction rate was sufficiently low that $\mathrm{LHCb}$ was able to operate with a very loose trigger which had almost $100 \%$ efficiency for all inelastic interactions.

Up to now, signs of open charm production have been searched using the high branching ratio decays $D^{0} \rightarrow K \pi$ and $D^{ \pm} \rightarrow K \pi \pi$. The reconstruction was based on flight distance, quality of the secondary vertex, impact parameter and on the ratio of the likelihoods for kaons and pions provided by the RICH detectors. The mass distributions for selected events are shown in Figure 1.

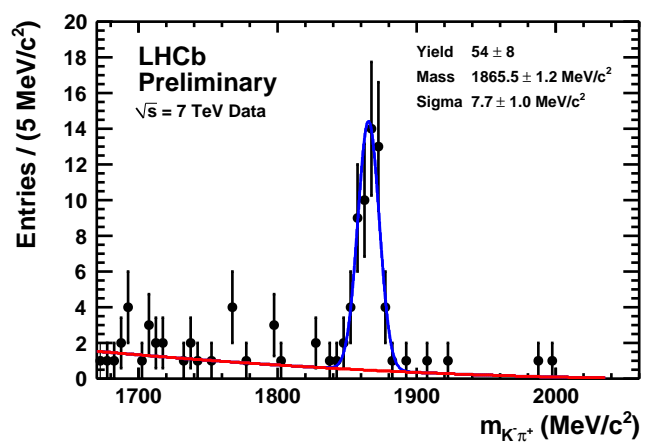

(a)

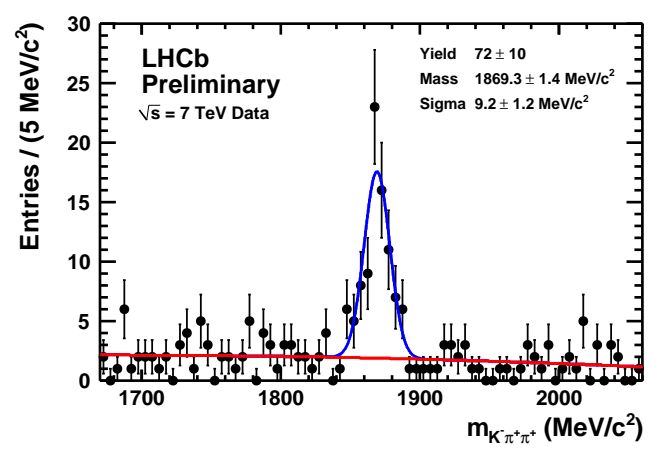

(b)

Figure 1: Invariant mass distributions for the decays $D^{0} \rightarrow K \pi$ (a) and $D^{ \pm} \rightarrow K \pi \pi$ (b).

In total, about 120 decays have been reconstructed with low background, from a sample corresponding to $\sim 120 \mu \mathrm{b}^{-1}$. These preliminary results, without a final tuning of the reconstruction algorithms, already provides mass values close to the world averages and widths similar to our Monte Carlo expectations.

\section{3. $J / \Psi$ production studies}

A long standing puzzle in the comprehension of the underlying production mechanism of quarkonia states was triggered by large discrepancies between QCD calculations and experimental measurements (see [4] and references in there). Measurements at the LHC may help in the construction of a clear and complete description of $J / \Psi$ production. LHCb will measure the $J / \Psi$ prompt and secondary $p_{T}$ differencial cross-section in 4 different $\eta$ bins covering the ranges $p \in[0,7] \mathrm{GeV} / \mathrm{c}$ and $\eta \in[3,5]$. This $\eta$ coverage is unique amongst the LHC experiments and is where the model predictions are less accurate.

The study of the cross-section has been performed using fully-simulated MC events with $\sqrt{s}=$ $14 \mathrm{TeV}$ [4]. The requirements used to select the $J / \Psi$ are essentially a good vertex formed by two 
good quality tracks well identified as muons. A detailed explanation of the muon identification procedure is given in Section 3.1. In order to separate the prompt (including feed-down from the decays of heavier charmonium states) from the secondary $J / \Psi$ component (produced in the decays of b-hadrons), a pseudo-proper time variable was defined as $t_{z}=\frac{d z}{p_{z}} \times m_{J / \Psi}$, where the flight distance and the $J / \Psi$ momentum are projected onto the beam axis. From the prompt component, shown in blue in the pseudo-propertime distribution of Fig. 2, the pseudo-propertime resolution can be extracted, which is convoluted with an exponential decay to reproduce the behaviour of the $J / \Psi$ coming from the B decays (in red). The long tail, due to the incorrect assignment of the primary vertex (PV), is calibrated with data, using a PV from a different event, as the PV and $J / \Psi$ vertex are uncorrelated. A fit with a Crystal-Ball function representing the signal and a linear function representing the background to the total mass distribution gives an estimated yield of $10^{9} \mathrm{~J} / \Psi$ with a S/B of $\sim 18$, corresponding to a luminosity of $1 \mathrm{fb}^{-1}$. With a combined fit of the mass and $t_{Z}$ distributions, the yields for the prompt and secondary components can be measured in each of the 28 bins, reproducing with good precision the input values given in the Monte Carlo sample. For $\sqrt{s}=7 \mathrm{TeV}$, a total yield of $\sim 3 \times 10^{6}$ is expected for an integrated luminosity of $5 \mathrm{pb}^{-1}$, providing measurements with a maximum statistical error below $10 \%$ in most $p_{T}$ and $\eta$ bins. Monte Carlo information will be used to correct for detector acceptance and trigger, reconstruction and selection efficiencies. By ignoring polarization, large systematic uncertainties (up to 25\%) have to be taken into account. In a second step, the measurement will be made also in separate bins of $\theta^{*}$, the angle between the $J / \Psi$ direction and the $\mu^{+}$momentum direction in the $J / \Psi$ rest frame. Similar studies will be performed with other charmonium states.

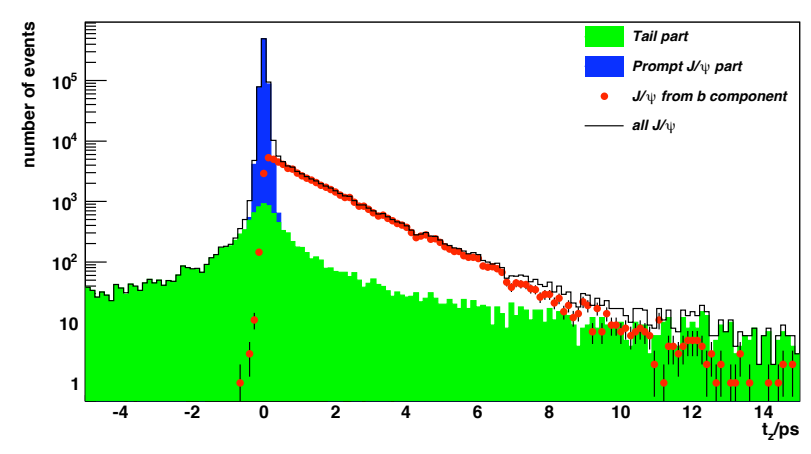

Figure 2: Distribution of the pseudo-propertime (LHCb simulation).

\subsection{Muon Identification Procedure}

The LHCb Muon System is composed of 5 tracking stations (M1-5) placed along the beam axis and separated by shields, consisting of the electromagnetic and hadronic calorimeter (between M1 and M2) and three $80 \mathrm{~cm}$ thick iron filters for a total absorber-thickness of 20 nuclear interaction lengths. Each station is subdivided in four regions covering increasing polar angles from the beam axis. These regions have dimensions and pad sizes which scales a factor two from one region to the next. 
The basic strategy to identify a muon in LHCb is based on three steps [5]. In the first one, each track reconstructed in both the VELO and Tracking System is linearly extrapolated, from a position after the magnet, to the 5 MS stations. Then a minimum number of stations is required to have at least one hit found within a field of interest (FOI) around the track extrapolation. The FOI in the $x$ and $y$ directions are functions of momentum defined for each of the 4 regions in the 5 MS stations and is dominated by the Coulomb Multiple Scattering for muons coming from the interaction point. For the beginning of data taking, a loose criterium to define a muon candidate was defined, requiring hits within the FOI in at least two stations among M2-M4, for tracks with momentum $p<6 \mathrm{GeV} / \mathrm{c}$, and at least three stations among M2-M5, for tracks with $p>6 \mathrm{GeV} / \mathrm{c}$. This requirement removes most of the background while keeping the muon efficiency above $95 \%$. A tighter muon candidate requirement is also built, requiring hits in M2 and M3 for tracks with $p \in[3,6] \mathrm{GeV} / \mathrm{c}$, in M2, M3 and M4 or M5 for $p \in[6,10] \mathrm{GeV} / \mathrm{c}$ and in all stations from M2 to M5 for tracks with $p>10 \mathrm{GeV} / \mathrm{c}$. The $p$ and $p_{T}$ distributions of tracks selected with these requirements in data are in very good agreement with the MC simulation. Finally, likelihoods are built for the hypotheses of being and not-being a muon. The likelihoods are based on the cumulative distributions of the average square distance (SD) from the closest hits to track extrapolation:

$$
S D=\frac{1}{N} \sum_{i=M 2, M 5}\left(\frac{x_{i}-x_{i}^{e x t}}{\Delta x_{i}}\right)^{2}+\left(\frac{y_{i}-y_{i}^{e x t}}{\Delta y_{i}}\right)^{2},
$$

where $x_{i}\left(y_{i}\right)$ is the hit $x(y)$ position in station $\mathrm{Mi}, x_{i}^{\text {ext }}\left(y_{i}^{\text {ext }}\right)$ is the $x(y)$ position of the track extrapolation to station $\mathrm{M} i$ and $\Delta x_{i}\left(\Delta y_{i}\right)$ is the detector pad size in the $x(y)$ direction for the hit. The distributions of this quantity are shown in Figure 3 (a), while the difference between the log of the likelihoods for the two hypotheses (DLL) are shown in Figure 3 (b). These plots are made with MC samples of true muons and non-muons. Excellent discrimination power is achieved. From MC simulation studies, a cut on the DLL providing $90 \%$ efficiency corresponds to a misidentification rate of $1 \%$ for tracks within the MS acceptance with $p>3 \mathrm{GeV} / \mathrm{c}$.
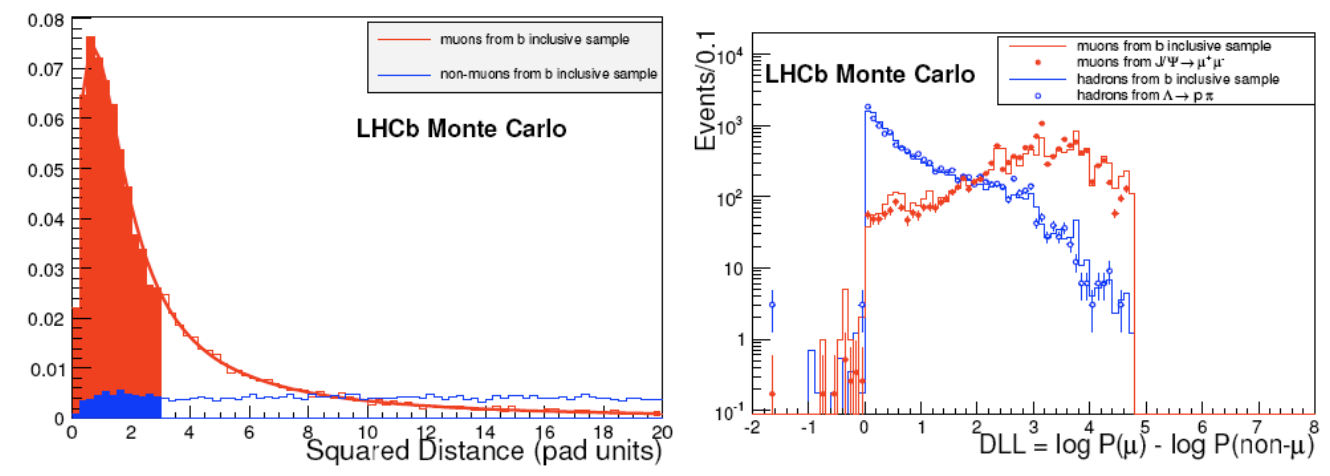

Figure 3: Distributions of the average square distance and the DLL for muon and non-muon tracks (MC).

All the parameters of the methods will be calibrated with data [6]. The FOI and muon likelihood will be calibrated with data using a pure sample of $J / \Psi \rightarrow \mu^{+} \mu^{-}$, where one of its daughters is used to tag the decay and the other one, remaining unbiased, is used for calibration and monitoring of the efficiency. The tag muon is selected using the Muon System information and the 
probe one is required to be compatible with a minimum ionising particle in the calorimeters. For the calibration of the non-muon likelihood, a sample of protons from $\Lambda \rightarrow p \pi$ decays will be used. Efficiency and proton, pion and kaon misidentification rates will be monitored using samples of $J / \Psi \rightarrow \mu \mu, \Lambda \rightarrow p \pi, K_{s}^{0} \rightarrow \pi \pi$ and $\phi \rightarrow K K$ decays, respectively.

\section{2 $\mathrm{J} /$ Psi reconstruction in 2010 data}

The invariant mass of opposite charge tracks have been plotted for tracks with $p>6 \mathrm{GeV} / \mathrm{c}$, satisfying the tight muon candidate requirements and making a good quality vertex. The result, corresponding to an integrated luminosity of $\sim 160 \mu \mathrm{b}^{-1}$, is shown in Fig. 4(a). A clear $J / \Psi$ peak can be seen. The central value is compatible with the world average and the width similar to MC expectations. No critical analysis of the yields have been done yet. The event display of one of the candidates with mass within the $J / \Psi$ peak is shown in Figure 4(b). The muon tracks are shown in magenta in the amplified view of the $y-z$ plane around the vertex position. The track in red makes a good vertex with the reconstructed $J / \Psi$ and satisfies requirements to be identified as a kaon. Moreover, the distance from the PV in the event is of the order of a few $\mathrm{mm}$. This event is the first $\mathrm{LHCb}$ candidate for the decay $B^{+} \rightarrow J / \Psi K^{+}$.

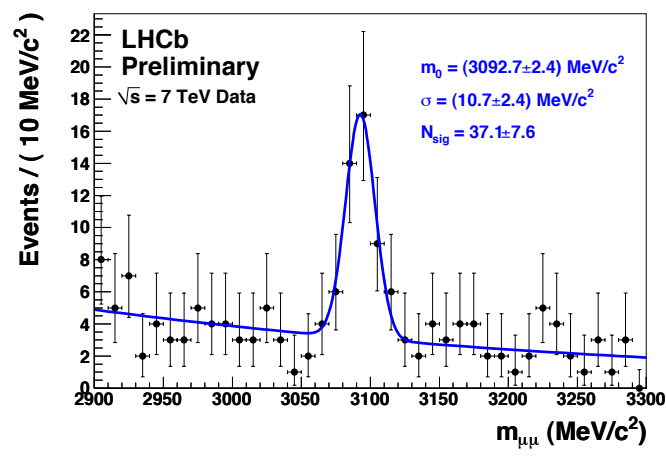

(a)

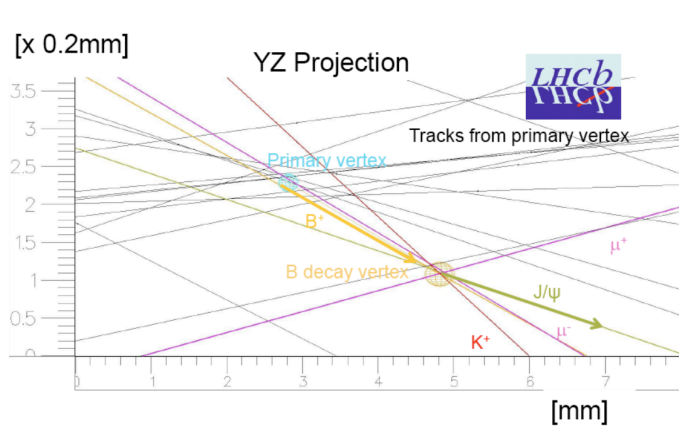

(b)

Figure 4: Di-muon invariant mass distribution (a) and $y-z$ view of the vertex region for one event with a $J / \Psi$ candidate (b).

\section{References}

[1] [LHCb Collaboration], Journal of Instrumentation, 3 (2008) S08005.

[2] M. Schiller, Results from LHCb: prompt VO production, in these proceedings, POS (DIS 2010) 137.

[3] D. Milanes, Charm physics at BaBar and Belle in these proceedings, PoS (Dis 2010) 166.

[4] Y. Gao et al., Measurement of the J/I meson production cross section in LHCb, LHCb-PUB-2010-011, (2010).

[5] G. Lanfranchi et al., The Muon Identification Procedure of the LHCb Experiment for the First Data, LHCb-PUB-2009-013 (2009).

[6] A. Sarti et al., Calibration Strategy and Efficiency Measurement of the Muon Identification Procedure at LHCb, LHCb-PUB-2010-002 (2010) 